\title{
The Ahmedabad Declaration, 2018: the family and diabetes
}

\author{
Banshi Saboo ${ }^{1}$ (D) Sanjay Kalra ${ }^{2}$ - Pratap Jethwani ${ }^{3} \cdot$ Rajeev Chawla $^{4}$ - B. M. Makkar ${ }^{5}$ - A. K. Das ${ }^{6}$ - O. P. Gupta ${ }^{7}$ \\ S. V. Madhu ${ }^{8}$ P. V. Rao ${ }^{9}$. Sanjeev Phatak ${ }^{10}$. Urman Dhruv ${ }^{11}$. Arun Kumar ${ }^{12}$
}

Received: 29 October 2018 / Accepted: 13 January 2019 / Published online: 8 February 2019

(C) Research Society for Study of Diabetes in India 2019

\begin{abstract}
The family is the basic social unit and should be taken as the basic unit of engagement and intervention in diabetes care as well. Family members not only contribute to diabetes care and support but are also prone to all effects arising from this. Shared genetic and environmental factors lead to a higher prevalence of diabetes in family members, and open a window of opportunity for diabetes prevention as well. The Ahmedabad Declaration of 2018, titled "The Family and Diabetes" has been released during the Annual Conference of the Research Society for Study of Diabetes India (RSSDI). It supports the theme of World Diabetes Day 2018-2019 and lends its voice towards strengthening the family in our quest for optimal diabetes control.
\end{abstract}

Keywords Community Compassion fatigue $\cdot$ Diabetes $\cdot$ Diabetes self-management education (DSME) Diabetes self-management support (DSMS) · Family · Society · Prevention

\section{Introduction}

The dictionary defines a family in various ways [1]. The noun family is a basic social group consisting of parents and their children. The word can be used as an adjective (diabetes is a family disease) and as an idiom (diabetes is common in women in the family way). The Institute for Patient- and Family-Centered Care (IPFCC) defines family members as two or more persons who are related in any way biologically, legally, or emotionally [2]. This allows a more inclusive concept of family, including nuclear, extended and kinship network members. It is also in concordance with Indian customs, where an entire village, community, caste, or tribe may be considered family.

Banshi Saboo

banshisaboo@hotmail.com

1 Diacare - Diabetes Care \& Hormone Clinic, 1 \& 2 Gandhi Park Society, Nr. Nehrunagar Cross Roads, Ambavadi, Ahmedabad, Gujarat 380015, India

2 Bharti Hospital, Karnal, Haryana, India

3 Jethwani Hospital, Rajkot, Gujarat, India

4 North Delhi Diabetes Centre, New Delhi, India

5 Dr. Makkar's Diabetes and Obesity Center, New Delhi, India

6 Pondicherry Institute of Medical Science, Puducherry, India
If family is the basic social unit, it stands to reason that it should be considered the basic unit of intervention in a syndrome such as diabetes, which has multiple social ramifications.

\section{The Indian family}

The attitudes, wishes, and needs of family members of persons living with diabetes were analyzed in the multinational second Diabetes Attitude, Wishes and Needs (DAWN2) study [3]. The DAWN2 study documented that there was a significant impairment of psychological well-being, diabetes-related distress, and negative impact on the life of these family members.

7 Dr. O P Gupta Clinic, Ahmedabad, Gujarat, India

8 University College Of Medical Sciences and GTB Hospital, New Delhi, India

9 Diabetes research Society, Hyderabad, Telangana, India

10 Vijayaratna Diabetes Diagnosis \& Treatment Center, India, Ahmedabad, India

11 HCG Hospital, Ahmedabad, Gujarat, India

12 Department of Community Medicine, Shaheed Hasan Khan Mewati, Government Medical College, Nalhar, Nuh, Haryana, India 
The frustration of family members in not being able to help their loved ones manage diabetes, and in dealing with hypoglycemia, was also observed. In spite of these challenge, most family members reported willingness to increase their involvement in diabetes care [4].

India was one of the 17 countries involved in DAWN2. Indians living with diabetes reported their family and "other people in the community," as being most supportive, as compared to peers from other countries. Indians with diabetes were the second most likely (after China) to have supportive friends, and third most likely (after China and Algeria) to encounter supportive people at work or school. This highlights the positive contribution of the family to diabetes care in India [5].

The Indian family does pay a high price for this contribution: the Indian family reported the third highest level of diabetes-related distress among all 17 countries (better only than Algeria and Turkey). Indian family members also reported a significant impact on their own leisure (rank 17/17), work or studies (17/17), physical health (15/17), emotional wellbeing (15/17), financial situation (11/17), and relationship with family/friends/nears (11/17). They were also the third most likely country to mention lack of any positive impact on their own health [5].

Yet, all these challenges did not prevent India from scoring the best on the chances of likely depression and third best on psychological well-being, among family members in all 17 DAWN2 countries. Another surprising effect was that Indian family members tended not to participate in formal diabetes education activities (rank 12/ 17). Even if they did do so, they rarely found these activities useful (17/17). Therefore, perhaps, they relied on other sources of knowledge and assistance (2/17) [5].

DAWN2 highlighted what has been termed "the Indian family paradox": though Indian family members had high levels of diabetes distress, they were able to maintain overall psychological well-being and were least likely to develop depression. Simultaneously, though they exhibited high levels of involvement in diabetes care, Indian family members were less likely to participate in formal educational activities and even less likely to find them useful. This, perhaps, prompts them to rely on other source of education, information, and support [5].

\section{The family as a cause}

The family acts as a cause of diabetes into two ways: through inheritance and through environmental factors. While type 2 diabetes is a multifactorial syndrome, a positive family history is noted in a significant proportion of seen in type 1 diabetes as well [6]. Monogenic forms of diabetes such as MODY (maturity onset diabetes of the young) are characterized by their strong family history [7]. Family history also informs the risk of developing specific complications, such as nephropathy and coronary artery disease, in diabetes.

Apart from the genetic aspect of causation, psychosocial and other environmental factors related to the family also play a role in etiopathogenesis of diabetes. Spouses of persons with diabetes have been documented to have a higher risk of developing the condition [8]. Shared dietary, physical activity, and stress-related and lifestyle-related characteristics may contribute to this.

\section{The family as a victim}

Though diabetes may be defined in terms of an individual's biomedical dysfunction, it impacts the life of the entire family. The management of diabetes may have to accept changes in their daily routine as well. Members of the family also have to shoulder responsibility for motivation and for care of the person with diabetes: this may lead to psychological stress, including compassion fatigue and burnout [9].

Diabetes-related expenditure can also affect the family economy and precipitate financial catastrophe [10]. Diversion of limited family funds, from "non-essential" items to diabetes care, may influence intra-family ties negatively. Family members who assist in tasks such as glucose monitoring and injection administration are prone to needle stick injuries and blood-borne disease [11].

\section{The family as therapy}

Family therapy is an accepted method of management in psychology. The same holds true for chronic diseases such as diabetes which has a strong psychosocial dimension [12]. The prevalence of diabetes in dysfunctional or broken families is documented to be high. Fostering of a strong and functional family unit can provide peace of mind and create a support system. This, in turn, facilitates the achievement of comprehensive biopsychosocial health while caring for persons with diabetes.

\section{The family as support}

The family acts as a great support in the management of diabetes (Table 1). A supportive family can join in diabetes education, assist in self-case and self-management activities, and encourage adherence to prescribed therapy. Multiple interventional trials have documented the benefit of family-based interventions often culture sensitized in improving diabetesrelated outcomes [13-15]. 
Table 1 The role of family in diabetes care

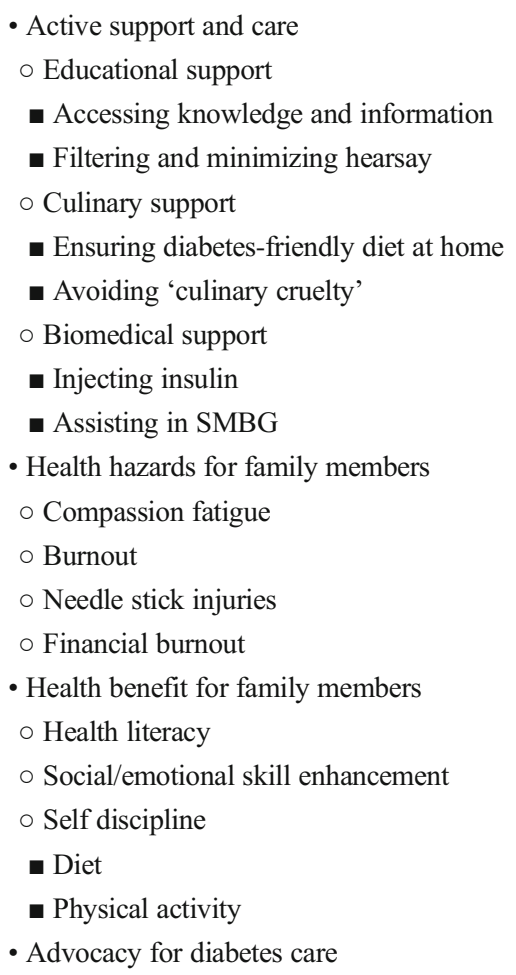

\section{The family as an opportunity}

The family of a person with diabetes represents a high-risk group of people from the perspective of development of diabetes [16]. This implies that such family should be viewed as a target for prevention of diabetes. This should be viewed as an opportunity for good health rather than as a cause for dejection. Apart from managing the individual with diabetes, the health care system should take the opportunity to screen his or her family members for diabetes, inculcate healthy lifestyle habits, and start timely pharmacotherapy if indicated. Regular screening and educational intervention for family members should be ensured.

\section{The family as an impediment}

Many cultures view chronic "disease," such as type 1 diabetes, as a social stigma or "shame," and ostracize individuals who develop the condition [17]. Family "honor," viewed as worthiness and respectability of the family, is perceived to be dependent on the health of its members. It is not uncommon to encounter situations where marital prospects of siblings of children living with type 1 diabetes have been affected by their "dishonorable" family history. Lack of diabetes literacy in the community can thus become an impediment to diabetes care [14].

\section{Limitations and caveats}

One must always be respectful of individual preferences. Some persons may not wish to disclose their diabetes status to a few or all family members for a variety of reasons. Selfdisclosure followed by family involvement should be encouraged but must not be forced, in such cases. Well-meaning, but ill-informed family members may share hearsay related to diabetes, which may lead to suboptimal health care seeking and acceptance. Family involvement, therefore, must be accompanied by proper medical education on an ongoing basis.

\section{The family as the future}

The diabetes epidemic is gradually converting to an endemic, which shows no signs of abating [18]. Some Indian states, such as Punjab, have a higher rate of diabetes than of impaired glucose tolerance [19], thus reporting a high-endemicity index. As health policymakers begin to understand the implications of this endemic, they will have to tailor their diabetescare strategies to include more sustainable family- and community-based interventions. This will help ensure that optimal results are achieved in prevention and treatment of diabetes. The family, therefore, should be, and will be, the basic unit of intervention in diabetes care.

\section{Resolution}

Keeping this in mind at Ahmedabad, we, as clinicians, researchers, and public health specialists, involved in diabetes care, resolve to:

- Spread awareness about the importance of the family in achieving optimal diabetes care.

- Support individuals with diabetes to communicate their needs and concerns to their family members.

- Strengthen the family in its capacity to prevent and manage diabetes and its complications.

- Sensitize health care professionals to actively involve family member in diabetes care

- Suggest to health policymakers to proactively create programs for education, screening, and treatment of family members of persons with diabetes.

- Solicit support from allied professions, including family medicine, community medicine/public health, and mental health, in improving activity targeting family members of persons with diabetes in health promotion activities.

- Start social marketing campaigns to improve treatment/ follow-up adherences and end social stigma or ostracization against persons with diabetes. 


\section{Action plan}

To achieve this resolution, we propose the following action plan for all diabetes care providers:

\section{At micro-level}

- Encourage persons with diabetes to be accompanied by family members and involve them in clinical consultations

- Provide diabetes screening and preventative facilities to family members

- Offer educational and psychological support to family members

\section{At meso-level}

- Form peer support groups of family members living with diabetes

- Create formal, professionally reviewed, educational tools for family members

- Create platforms where family members can articulate their wishes, needs, and concern to diabetes care providers

\section{At macro-level}

- Consider a national or state level promotive health program focusing on family members of persons with diabetes

- Use family members as advocates of timely diabetes care

- Involve family members of persons with diabetes in creation of patient-oriented guidelines and recommendations

\section{Summary}

The Ahmedabad Declaration of 2018 is issued on the occasion of the Annual Conference of the Research Society for Study of Diabetes in India (RSSDI). The Ahmedabad Declaration supports the efforts of the International Diabetes Federation (IDF), World Diabetes Day 2018-2019 with the theme "The Family and Diabetes", over the next year, we hope that this Declaration will facilitate active involvement of the family in diabetes care, as well as encourage actionable focus on the health of these family members as well.

Publisher's note Springer Nature remains neutral with regard to jurisdictional claims in published maps and institutional affiliations.

\section{References}

1. Family. Available at: https://www.dictionary.com/browse/family. Last Accessed on 25 Oct 2018

2. Patient- and family-centered care. Available at: http://www.ipfcc. org/about/pfcc.html. Last Accessed on 25 Oct 2018.

3. Peyrot M, Burns KK, Davies M, Forbes A, Hermanns N, Holt R, et al. Diabetes attitudes wishes and needs 2 (DAWN2): a multinational, multi-stakeholder study of psychosocial issues in diabetes and person-centred diabetes care. Diabetes Res Clin Pract. 2013;99(2):174-84.

4. Kovacs Burns K, Nicolucci A, Holt RI, Willaing I, Hermanns N, Kalra S, et al. DAWN2 study group. Diabetes Attitudes, Wishes and Needs second study (DAWN2 ${ }^{\mathrm{TM}}$ ): cross-national benchmarking indicators for family members living with people with diabetes. Diabet Med. 2013;30(7):778-88.

5. Kalra S, John M, Baruah MP. The Indian family fights diabetes: results from the second diabetes attitudes, wishes and needs (DAWN2) study. J Soc Health Diabetes. 2014;2(1):3.

6. McCarthy MI. Genomics, type 2 diabetes, and obesity. N Engl J Med. 2010;363(24):2339-50.

7. Mohan V, Radha V, Nguyen TT, Stawiski EW, Pahuja KB, Goldstein LD, et al. Comprehensive genomic analysis identifies pathogenic variants in maturity-onset diabetes of the young (MODY) patients in South India. BMC medical genetics. 2018;19(1):22.

8. Khan A, Lasker SS, Chowdhury TA. Are spouses of patients with type 2 diabetes at increased risk of developing diabetes? Diabetes Care. 2003;26(3):710-2.

9. Kalra S, Sridhar GR, Balhara YP, Sahay RK, Bantwal G, Baruah MP, et al. National recommendations: psychosocial management of diabetes in India. Indian journal of endocrinology and metabolism. 2013;17(3):376-95.

10. Sahay R, Baruah MP, Kalra S. Health economics in India: the case of diabetes mellitus. Indian J Endocrinol Metab. 2014;18(2):135-7.

11. Kalra S, Gupta Y. Professional hazards of diabetes care professionals. JPMA J Pak Med Assoc. 2016;66(4):483-4.

12. Powers MA, Bardsley J, Cypress M, Duker P, Funnell MM, Fischl $\mathrm{AH}$, et al. Diabetes self-management education and support in type 2 diabetes: a joint position statement of the American Diabetes Association, the American Association of Diabetes Educators, and the Academy of Nutrition and Dietetics. The Diabetes Educator. 2017;43(1):40-53.

13. Ahmed Z, Yeasmeen F. Active family participation in diabetes selfcare: a commentary. Diabetes Manag. 2016;6(5):104-7.

14. Mayberry LS, Osborn CY. Family involvement is helpful and harmful to patients' self-care and glycemic control. Patient Educ Couns. 2014;97(3):418-25.

15. Baig AA, Benitez A, Quinn MT, Burnet DL. Family interventions to improve diabetes outcomes for adults. Ann N Y Acad Sci. 2015;1353(1):89-112.

16. American Diabetes Association. 2. Classification and diagnosis of diabetes: standards of medical care in diabetes-2018. Diabetes Care. 2018;41(Supplement 1):S13-27.

17. Liu NF, Brown AS, Folias AE, Younge MF, Guzman SJ, Close KL, et al. Stigma in people with type 1 or type 2 diabetes. Clinical Diabetes. 2017;35(1):27-34.

18. Kalra S, Kumar A, Jarhyan P, Unnikrishnan AG. Endemic or epidemic? Measuring the endemicity index of diabetes. Indian $\mathrm{J}$ Endocrinol Metab. 2015;19(1):5-7.

19. Anjana RM, Deepa M, Pradeepa R, Mahanta J, Narain K, Das HK, et al. Prevalence of diabetes and prediabetes in 15 states of India: results from the ICMR-INDIAB population-based cross-sectional study. Lancet Diabetes Endocrinol. 2017;5(8):585-96. 